\title{
Three Types of Semiosis
}

\author{
Marcello Barbieri
}

Received: 30 June 2008 / Accepted: 14 October 2008 /

Published online: 17 December 2008

(C) Springer Science + Business Media B.V. 2008

\begin{abstract}
The existence of different types of semiosis has been recognized, so far, in two ways. It has been pointed out that different semiotic features exist in different taxa and this has led to the distinction between zoosemiosis, phytosemiosis, mycosemiosis, bacterial semiosis and the like. Another type of diversity is due to the existence of different types of signs and has led to the distinction between iconic, indexical and symbolic semiosis. In all these cases, however, semiosis has been defined by the Peirce model, i.e., by the idea that the basic structure is a triad of 'sign, object and interpretant', and that interpretation is an essential component of semiosis. This model is undoubtedly applicable to animals, since it was precisely the discovery that animals are capable of interpretation that allowed Thomas Sebeok to conclude that they are also capable of semiosis. Unfortunately, however, it is not clear how far the Peirce model can be extended beyond the animal kingdom, and we already know that we cannot apply it to the cell. The rules of the genetic code have been virtually the same in all living systems and in all environments ever since the origin of life, which clearly shows that they do not depend on interpretation. Luckily, it has been pointed out that semiosis is not necessarily based on interpretation and can be defined exclusively in terms of coding. According to the 'code model', a semiotic system is made of signs, meanings and coding rules, all produced by the same codemaker, and in this form it is immediately applicable to the cell. The code model, furthermore, allows us to recognize the existence of many organic codes in living systems, and to divide them into two main types that here are referred to as manufacturing semiosis and signalling semiosis. The genetic code and the splicing codes, for example, take part in processes that actually manufacture biological objects, whereas signal transduction codes and compartment codes organize existing objects into functioning supramolecular structures. The organic codes of single cells appeared in the first three billion years of the history of life and were involved either in manufacturing semiosis or in signalling semiosis. With the origin of animals, however, a third type of semiosis came into being, a type that can be referred to as
\end{abstract}

M. Barbieri $(\bowtie)$

Dipartimento di Morfologia ed Embriologia, Via Fossato di Mortara 64, 44100 Ferrara, Italy

e-mail: brr@unife.it 
interpretive semiosis because it became closely involved with interpretation. We realize in this way that the contribution of semiosis to life was far greater than that predicted by the Peirce model, where semiosis is always a means of interpreting the world. Life is essentially about three things: (1) it is about manufacturing objects, (2) it is about organizing objects into functioning systems, and (3) it is about interpreting the world. The idea that these are all semiotic processes, tells us that life depends on semiosis much more deeply and extensively than we thought. We realize in this way that there are three distinct types of semiosis in Nature, and that they gave very different contributions to the origin and the evolution of life.

Keywords Semiosis · Biosemiotics · Interpretation · Organic codes · Peirce

\section{Introduction}

The existence of the genetic code in every living cell suggests that the cell is a semiotic system, i.e., a system based on signs, but this conclusion has not been accepted by modern biology. The genetic code is normally described as a set of mapping rules, like those of the Morse code, for example, and this suggests a certain similarity with the codes of culture, but nothing more than that. According to modern biology, signs and meanings do not exist at the molecular level, and this implies that the genetic code is a metaphorical entity, not a real code.

Here it is shown that these widespread assumptions are wrong. More precisely, it will be shown (1) that signs and meaning do exist at the molecular level, and (2) that the genetic code is very much different from the Morse code. In order to discuss these points, however, it is necessary to be clear about the terms that we are using, and in particular about the definition of semiotic system, so let us make a little premise.

Semiosis is usually described as the production of signs, but this definition is too restrictive because signs are always associated with other entities. A sign, to start with, is always linked to a meaning because it is only when we give a meaning to something, that that something becomes a sign for us. Sign and meaning, in other words, cannot be taken apart because they are the two sides of the same coin. Semiosis, therefore, is not just the production of signs; it is the production of signs and meanings together. The result is that a system of signs, i.e., a semiotic system, is always made of at least two distinct worlds: a world of entities that we call signs and a world of entities that represent their meanings.

The link between sign and meaning, in turn, calls attention to a third entity, i.e., to their relationship. A sign is a sign only when it stands for something that is other than itself, and this otherness implies at least some degree of independence. It means that there is no deterministic relationship between sign and meaning. Different languages, for example, give different names to the same object precisely because there is no necessary connection between names and objects. A semiotic system, therefore, is not any combination of two distinct worlds. It is a combination of two worlds between which there is no necessary link, and this has an extraordinary consequence. It implies that a bridge between the two worlds can be established only by conventional rules, i.e., by the rules of a code. This is what qualifies the semiotic systems, what makes them different from everything else: a semiotic system is a 
system made of two independent worlds that are connected by the conventional rules of a code. A semiotic system, in conclusion, is necessarily made of at least three distinct entities: signs, meanings and code.

Signs, meanings and codes, however, do not come into existence of their own. There is always an 'agent' that produces them, and that agent can be referred to as a codemaker because it is always an act of coding that gives origin to semiosis. In the case of culture, for example, the codemaker is the human mind, since it is the mind that produces the mental objects that we call signs and meanings and the conventions that link them together. We come in this way to a general conclusion that can be referred to as 'the code model of semiosis': a semiotic system is a triad of signs, meanings and code that are all produced by the same agent, i.e., by the same codemaker (Barbieri 2003, 2008).

This conclusion is highly relevant to our problem because it tells us precisely what we need to prove in order to show that the cell is a semiotic system. We need to prove that in every living cell there are four distinct entities: signs, meanings, code and codemaker.

\section{The Defining Feature of Signs and Meanings}

A semiotic system is made of signs, meanings, code and codemaker, and we know that there is a genetic code in protein synthesis. We also know that proteins are made by a system of ribonucleoproteins that is the physical seat of the genetic code and that functions therefore as the codemaker of the cell (Barbieri 2003). This tells us that every living cell does have a genetic code and a codemaker. But what about the other two entities? Can we say that there are also signs and meanings at the molecular level? Can these entities exist in the cell? In order to answer this question, let us examine first the traditional signs and meanings of culture and see if they have a qualifying feature that can be extended to the molecular level.

The signs and meanings that we are familiar with are often the mental representations of objects or events of the physical world. A sign, for example, can be a spoken word and its meaning can be a mental image. The mental image of an object is normally evoked by different words in different languages, and this clearly shows that mental sounds and mental images are separable. When they are separated, however, they no longer function as signs and meanings. To a nonEnglish speaker, for example, a word like 'twitch' may have no linguistic meaning and in this case it would be just a sound, not a sign. There is no contradiction therefore in saying that signs and meaning are distinct mental objects and that they cannot be taken apart, because when they are taken apart they simply stop functioning as signs and meanings.

This makes us understand an extremely important feature of semiosis. It tells us that a mental sign, or a mental meaning, is never an intrinsic property of a mental object. It is something that the mind can give to a mental object and that the mind can take away from it. One could object that terms like mental signs and mental objects are a clear case of mentalism, and this is no longer the received view, today. The above conclusion, however, remains valid even if we accept that the mind is but a product of the brain and that sounds and images are just the results of neuron 
firings. Even in this case, the link between the neuron firings that produce the signs and meanings of a language are based on the rules of a code and are totally dependent upon the 'agent' of that code, i.e., upon the codemaker of the system.

Signs and meanings simply do not exist without a codemaker and outside a codemaking process. The codemaker is the agent of semiosis, whereas signs and meanings are its instruments. We conclude therefore that signs and meanings are totally dependent on codemaking, i.e., they are codemaker-dependent entities. This is the qualifying feature that we were looking for, because it is completely general and can be applied to all systems. We can say therefore that signs and meanings exist at the molecular level, and in particular in protein synthesis, only if we prove that in protein synthesis there are codemaker-dependent entities.

\section{The Sequences of Genes and Proteins}

All biochemistry textbooks tell us that there is a genetic code in protein synthesis, but none of them mentions the existence of signs and meanings. At first sight, in fact, these entities do not seem to exist at the molecular level. The translation apparatus can be regarded as a codemaker because it is the seat of the code that creates a correspondence between genes and proteins, but these molecules appear to have only 'objective' chemical properties, not the 'codemaker-dependent' properties that define signs and meanings. A messenger RNA, for example, appears to be a unique and objective sequence of molecules, but let us take a closer look.

A messenger RNA is certainly a unique and objective chain of nucleotides but in no way it is a unique sequence of codons because different codemakers could scan it in different ways. If the nucleotides were scanned two-by-two, for example, the sequence of codons would be totally different. The same chain of nucleotides, in other words, can give origin to many sequences of codons, and it is always the codemaker that determines the sequence because it is the codemaker that defines the codons. A linear sequence of codons, in short, does not exist without a codemaker and outside a codemaking process. It is totally dependent on codemaking and is therefore a codemaker-dependent entity, which is precisely what a sign is.

In the same way, the linear sequence of amino acids that is produced by the translation apparatus is also a codemaker-dependent entity, because only a codemaker can produce it. Any spontaneous assembly of amino acids would not make linear chains, and above all it would not arrange the amino acids in a specific order. Specific linear sequences of amino acids can be produced only by codemakers, but different codemakers would arrange the amino acids in different ways, which shows that the sequence of a protein is only one of the many possible 'meanings' that could be given to a string of nucleotides.

The sequence of a gene and the sequence of a protein, in conclusion, are not objective properties of those molecules. They are codemaker-dependent properties because they do not exist without a codemaking process, and because they would be different if the codemaker had a different structure. The sequences of genes and proteins, in short, have precisely the characteristics that define signs and meanings. They are codemaker-dependent entities made of organic molecules and are therefore organic signs and organic meanings. All we need to keep in mind is that signs and 
meanings are mental entities when the codemaker is the mind, but they are organic entities when the codemaker is an organic system (Barbieri 2003).

We reach in this way the conclusion that every living cell contains all four components of semiosis (signs, meanings, code and codemaker) and is therefore a real semiotic system.

\section{Two Types of Signs}

Signs have been divided since antiquity into two great classes that are traditionally represented by symbols and symptoms. Augustine of Hippo (389ad) called them signa data and signa naturalia, a distinction that continues to these days under the terms of conventional signs and natural signs (Deely 2006; Favareau 2007). The conventional signs are those where there is no physical relationship between signifiers and meanings and a connection between them can be established only by arbitrary rules, i.e., by conventions. Words, for example, are signs (because they 'stand for' the named entities) and are conventional signs because they are not determined by the characteristics of the named entities. In the same way, there is no necessary connection between symbols and the entities that they stand for (between a flag and a country, for example).

In natural signs, by contrast, a physical link is always present between signifier and signified. Typical examples are the symptoms that doctors use to diagnose illnesses (spots on the skin, a fever, a swollen area, etc.), as well as a variety of cues (smoke as sign of fire, odours as signs of food, footprints as signs of organisms, etc.). In all these cases there is a physical relationship between the visible signs and the invisible entities that they point to, and yet the relationship is underdetermined, so much so that it takes a process of learning and an act of interpretation to establish it. The diagnosis of an illness from symptoms, for example, is always an interpretative exercise, and even simple associations, such as those between clouds and rain, depend upon processes of learning and memory.

At the molecular level, we have seen that in protein synthesis a sequence of nucleotides is used as a sign, by a codemaker, to produce a sequence of amino acids according to the rules of the genetic code. In that case, there is no necessary connections between the components of the two molecules and the sequence of nucleotides is used therefore as a conventional organic sign, i.e., as an organic symbol.

A sequence of nucleotides, however, can also be used by a copymaker to produce a complementary copy of itself, and in that case the relationship between the two sequences is no longer established by a code but by direct physical interactions between complementary surfaces. These interactions, however, occur between very small regions of the molecules, and that means that the first sequence provides only a limited number of physical determinants for the second. The first sequence, in other words, does have a physical relationship with the second, but such relationship is undetermined and represents therefore only a 'cue', i.e., a natural sign, for the second.

We conclude that the distinction between natural and conventional signs exists also at the molecular level, and represents in fact a divide between two very different types of molecular processes. Sequences of nucleotides are used as natural signs in molecular copying, and as conventional signs in molecular coding. The replication of 
genes, in other words, is based on natural organic signs, whereas the synthesis of proteins is based on conventional organic signs.

\section{The Concept of Manufacturing Semiosis}

The idea that the genetic code is a set of mapping rules like the Morse code, for example, is still very popular today, and yet it is only a superficial analogy. It is true in fact that the genetic code and the Morse code can both be described by a 'transformation matrix' or a 'conversion table', but if we take a closer look we discover that there are very substantial differences between them.

(a) One is the fact that the Morse code is perfectly reversible, or invertible. It transforms the letters of the alphabet into dots and dashes and, vice versa, dotsand-dashes into letters of the alphabet. The same applies to many other cultural codes, for example to the codes that allow us to translate a text from English to Chinese and from Chinese to English, or to transform written words into sounds and sounds into written words. Nothing of the kind takes place in the cell. The genetic code is absolutely irreversible, or non-invertible. It is a process that goes from genes to proteins and absolutely not viceversa. The reverse transformation is not just avoided, it is physically impossible.

(b) Another difference is that the messages written in Morse are perfectly equivalent to those of the Alphabet world. They carry exactly the same information and are simply a different way of expressing the same reality. The Morse code, in short, transforms a world of entities into a world of equivalent entities. In the case of the genetic code, instead, the situation is totally different. Genes and proteins are not at all equivalent objects, they belong to completely different worlds.

We reach in this way two important conclusions: (1) one is that protein synthesis is a process of semiosis because it is based on code and signs, (2) the other is that protein synthesis is not just a semiosis, but a manufacturing semiosis, i.e., a type of semiosis whose purpose is to produce objects that cannot come into existence in any other way. For a long time it has been assumed that the function of semiosis is to interpret the world, and this is undoubtedly true in some cases, but it is not the whole truth. We must acknowledge that there is another type of semiosis whose function is not to interpret the world of life but to manufacture it, to bring its objects into existence.

\section{The Concept of Signalling Semiosis}

Protein synthesis is the first example of manufacturing semiosis in the history of life, but not the only one. Another example if splicing, the process that cuts away RNAs pieces from primary transcripts and assembles the remaining pieces into messenger RNAs. Splicing is closely similar to protein synthesis because the splicing bodies, known as spliceosomes, are huge molecular machines like ribosomes, and employ small molecular structures, known as snRNAs or snurps, which are like tRNAs. The 
similarity, however, goes much deeper than that, because the snRNAs have properties that fully qualify them as adaptors, and the presence of adaptors is the experimental proof that a process is based on codes (Barbieri 2003). Splicing is therefore a semiosis, and more precisely a manufacturing semiosis, because it actually brings into existence objects that cannot be produced in any other way.

There are however other types of semiosis that do not have a manufacturing function. Signal transduction, for example, is a semiosis because it creates a correspondence between first and second messengers by means of receptor molecules that have all the defining features of adaptors, but it is not a manufacturing semiosis because the second messengers already exist in the cell and are not manufactured by the transduction process. Signal transduction has the function to create a specific signalling association between first and second messengers and is therefore a semiosis that can be referred to as signalling semiosis.

Another example of signalling semiosis is given by the compartment codes of the eukaryotic cells. In this case too we recognize the existence of semiosis by the presence of adaptors and we realize that its function is to create specific signalling associations between pre-existing objects, not to bring these objects into existence. The function of signalling semiosis is therefore totally different from that of manufacturing semiosis, but it is equally fundamental to life. Manufacturing semiosis is about generating objects, whereas signalling semiosis is about organizing them into functioning structures.

Proteins, for example, are produced by manufacturing semiosis, but in order to organize them into supramolecular structures like cell membrane, cytoskeleton and intracellular compartments, we need other types of processes, and the existence of adaptors proves that many of these processes are also based on semiosis. Manufacturing semiosis and signalling semiosis, in other words, have very different functions but they are both essential to life and represent therefore complementary processes.

The presence of the two types of semiosis can also be recognized at many other levels of organization. Mental objects, for example, are brought into existence by manufacturing mental processes (Barbieri 2006), and once in existence they are organized into mental structures by signalling processes. Manufacturing semiosis and signalling semiosis, in short, exist and have complementary functions at many levels of organizations and must have evolved together again and again in the history of life.

\section{The Origin of Subjectivity}

Single cells are capable of extremely sophisticated interactions with the environment, to the point that one is easily tempted to regard them as miniature animals, but in reality there is a very substantial difference between those two forms of life. In order to understand why, we have to go back to the concept of meaning, and to a classic distinction introduced by Gottlob Frege.

The meaning of a sign is, by definition, what the sign stands for, but this can be of two very different types. A sign can stand for an object that is 'external' or 'internal' to the system. The difference between these two types of meaning was outlined by Frege with an example that is reported in virtually every discussion of this issue. 
Frege pointed out that the terms "Morning Star" and "Evening Star" both indicate the planet Venus, and have therefore the same 'external' meaning, but very different 'internal' meanings. The "Evening Star" is visible at dusk and was related to end-ofthe-day events, whereas the "Morning Star" was related to the rise of the sun and to the beginning of a new day (Frege 1892).

In order to differentiate between external and internal meanings, Frege gave them two different names, and called them respectively 'reference' and 'sense'. Other authors have adopted a different terminology, and have used terms like 'extension' and 'intension', or 'denotation' and 'connotation'. The point, however, is the same. All entities associated to signs by conventions qualify as meanings, but some may refer to outside objects (reference) and others to internal entities (sense).

After Frege, it has been taken almost for granted that all signs have both types of meanings, but this is not always the case. Proteins, for example, are built according to the genetic code, and this is a set of exclusively internal rules. Proteins do not have any other meanings in addition to those that come from genes, and this implies that the apparatus of protein synthesis is a system of signs which have sense but not reference, i.e., signs which have internal meanings but not external ones. This lack of reference in protein synthesis may appear surprising, at first, but it does have a natural explanation.

The human mind (or brain) receives signals from the environment and uses them to produce internal representations of the world, representations that are the external meanings, the reference, that the mind (or the brain) associates to the incoming signals. The mind, on the other hand, is also giving internal meanings to signs, and must integrate them with the external meanings in order to allow the body to react to the incoming signals. This makes us understand why the mind (or the brain) must necessarily use two types of meanings. If the mind could act directly on the environmental signals, there would be no need of external meanings, but that is not what happens. The mind can only act on representations of the world and that is why it must use signs which have both internal and external meanings, i.e., sense and reference.

A single cell, on the other hand, does not build internal representations, so it does not need two types of meaning. Single cells produce their components and interact with the world with a variety of codes which are based exclusively on internal rules, i.e., on sense, not on reference. They 'decode' the world with sophisticated sets of conventions, but do not build internal images of the world. They are sensitive to light, but do not 'see'; they react to sounds but do not 'hear'; they detect hormones but do not 'smell' and do not 'taste' them. It takes the cooperation of many cells which have undertaken specific processes of differentiation to allow a system to see, hear, smell, taste, etc., so it is only multicellular creatures that can have these experiences.

Only animals, in short, build internal representations of the world and only these representations allow them to perceive and to feel. But perceptions and feelings are precisely what we call subjective experiences, so it is only animals that have what we call 'subjectivity'.

The evolution from single cells to animals was far more than an increase in growth and complexity. It was a true macroevolutionary event that gave origin to absolute novelties, to entities that had never existed before such as perceptions and feelings. Subjectivity had an origin and a history, like everything else in life, and could appear only in multicellular systems. That is what really divides animals from single cells. 


\section{The Concept of Interpretive Semiosis}

The organic codes of single cells appeared in the first three billion years of the history of life, during the period of cellular evolution, and were involved either in manufacturing semiosis or in signaling semiosis. With the origin of animals, however, a third type of semiosis came into being, a type that will be referred to as interpretive semiosis because it became specifically involved in the process of interpretation.

The first animals were probably organisms whose behaviour was almost entirely programmed by genes, but there is a limit to the number of instructions that can be carried in a genome, and that set the stage for the evolution of a new type of behaviour. Since the number of hard-wired responses could not grow indefinitely, animals started resorting to processes of learning in order to increase their behavioral repertoire.

Learning how to respond to a signal, on the other hand, means learning how to interpret that signal, and this is essentially a context-dependent process. At the same time, learning requires a memory where the results of experience are accumulated, which means that interpretation is also a memory-dependent process. Animals, in short, became increasingly capable of interpreting the world, and this is a process that does not arise automatically from organic codes. Interpretation is essentially what Peirce called an "abduction", a process that is neither induction nor deduction, but a "rule of thumb" way of creating a link between inputs and outputs (Peirce 1906). Interpretation, on the other hand, requires a comparison of incoming signals with an internal reference, and takes place therefore only in systems that build internal representations of the world. Interpretation, in short, is a semiosis because it is a process that gives meaning to signs, but it is a new type of semiosis because it is dependent on learning, memory and context, and probably evolved only in animals. Language came after another 500 million years and apparently evolved only in our species.

The history of semiosis, in conclusion, was a process that started with contextfree codes and produced codes that were more and more context-dependent. Today, our cultural codes are so heavily dependent on context that we can hardly imagine semiosis without interpretation, and yet these are distinct processes and we need to keep them apart if we want to understand their origin and their evolution in the history of life.

\section{The Peirce Model of Semiosis}

The idea that semiosis is based on interpretation was proposed by Peirce with the model that a sign is always a triadic relationship between a representamen, an object and an interpretant (Peirce 1906), but it was Thomas Sebeok who put this model at the centre of semiotics. In 1963, Sebeok challenged the century old belief that only man is a semiotic animal and proposed that animals too communicate by signs. He gave the name of zoosemiotics to the study of animal semiosis and adopted the model of Peirce because it is precisely the animals' ability to interpret the world that proves the existence of semiosis in them (Sebeok 1963).

This concept was later extended beyond the animal world in various stages. In 1981, Martin Krampen argued that plants too engage in semiosis, and in 1988, Sorin Sonea proposed that semiosis goes on even in the bacterial world (Krampen 1981; 
Sonea 1988). Still in 1988, Giorgio Prodi suggested that a primitive form of semiosis exists also at the molecular level and called it protosemiosis, or natural semiosis (Prodi 1988). The word zoosemiotics became increasingly inadequate and eventually Sebeok replaced it officially with 'biosemiotics'.

The extension of semiosis to all living creatures did not ignore the diversities between them and it was acknowledged that there are different types of semiosis in different taxa. Plant semiosis, for example, is distinct from animal semiosis and both of them from the semiosis of fungi, protists and bacteria. Despite the differences, however, they are all semiotic processes, and this allows us to the conclude that semiosis exists in all living systems.

This is the essence of biosemiotics and all biosemioticians accept it, but Sebeok's proposal was not limited to that. He insisted that what all living systems have in common is not just semiosis but interpretive semiosis. This amounts to saying that the Peirce model applies not only to animals but to all living creatures, i.e., that all types of semiosis are based on interpretation. Sebeok expressed this concept in no uncertain terms by declaring that: "there can be no semiosis without interpretability" (Sebeok 2001). This conclusion was also formally expressed in an a treatise that defined semiosis in unmistakably Peircean terms, i.e., in terms of interpretation:

"We stipulate that the following is a necessary and sufficient condition for something to be a semiosis: A interprets B as representing C" (Posner et al. 1997).

By the 1990s, in short, the Peirce model of semiosis had become almost universally accepted, and it was taken virtually for granted that all types of semiosis are based on interpretation.

\section{Three Types of Semiosis}

The Peirce model is undoubtedly valid for animals, but its extension to other living creatures is much more problematic and we definitely cannot apply it to the cell. This is because the rules of the genetic code have been virtually the same in all living systems ever since the origin of life, which clearly shows that they do not depend on interpretation.

The concept of semiosis, on the other hand, can de defined without any reference to interpretation because there is no necessary link between them. It can be defined exclusively in terms of coding, and in this form it is immediately applicable to the cell. According to the 'code model', a semiotic system is made of signs, meanings and coding rules, all produced by the same codemaker. In the case of the cell, the translation apparatus is the codemaker, i.e., the seat of the genetic code, the transferRNAs are the adaptors that implement the rules of the code, and the sequences of codons and amino acids have the defining features of signs and meanings because they are codemaker-dependent entities. This tells us that the cell is indeed a semiotic system, but its semiosis is based on coding, not on interpretation (Barbieri 2008).

It may be pointed out that the traditional concept of interpretation could be 'generalized' when we extend it from animals to other living creatures. Couldn't we say, for example, that an act of coding is also an act of interpretation? In principle, of 
course, we could, but there would be a price to pay, and there are at least two consequences that should warn us against it.

(1) If we generalize the concept of interpretation we can easily end up with a concept that is applicable virtually to everything. Edwina Taborsky, for example, has concluded that any function $f(x)=y$ is an act of interpretation whereby the function ' $f$ ' interprets ' $x$ ' as representing ' $y$ ' (Taborsky 1999, 2002). In this way all physical laws expressed by functions like $f(x)=y$ would be processes of interpretation and therefore acts of semiosis.

The important point is that Peirce himself embraced the view that semiosis exists everywhere in the Universe, and this is an idea which has enormous consequences. First, it implies that semiosis originated either in the Big Bang or inside the giant stars that produced the atoms of the universe, and then it implies that the laws of physics undergo changes that turn them into 'habits' of nature, an idea that requires a completely new paradigm for which we have not a shred of evidence. We realize in this way that a generalized concept of interpretation gives us a pansemiotic view of Nature, and that this view is in sharp contrast with modern physics. If we want to keep our present understanding of the laws of physics, therefore, we must also keep the traditional concept of interpretation which is applicable only to animal life.

(2) The idea that semiosis is based on interpretation not only in animals but in all living creatures, implies that semiosis is always an 'interpretive' process, that semiosis is only a means of interpreting the world, and this is a very severe limitation, because it means that life gets only a small contribution from semiosis.

Life is essentially about three things: (1) it is about manufacturing objects, (2) it is about assembling objects into functioning structures, and (3) it is about interpreting the world. The idea that these are all semiotic processes, tells us that life depends on semiosis much more deeply and extensively than we thought on the basis of the Peirce model. The Peirce model of semiosis, in other words, is too limited, too restrictive. There are three distinct types of semiosis in Nature and interpretive semiosis is only one of them.

We know that Peirce too spoke of three types of semiosis, based on Icons, Indexes and Symbols, but in his scheme they were all dependent on interpretation and were therefore different types of interpretive semiosis. In reality, even this is an unnecessary restriction. Icons, indexes and symbols can exist also in manufacturing semiosis and in signalling semiosis, because they too do not necessarily depend on interpretation.

We need, in conclusion, to go beyond the Peirce model not because it is wrong but because it is too restrictive. It is limited to the processes of interpretation and these account only for a small fraction of life. It is about time that we come to terms with the existence of manufacturing semiosis and signalling semiosis in all forms of life, and realize that they actually are the preconditions for the existence of interpretive semiosis.

\section{References}

Augustine of Hippo (389ad). De Doctrina Christiana. In W. M. Green (Ed.), Sancti Augustini Opera. Vienna: CSEL 801963. 
Barbieri, M. (2003). The organic codes. An introduction to semantic biology. Cambridge, UK: Cambridge University Press.

Barbieri, M. (2006). Semantic biology and the mind-body problem: the theory of the conventional mind. Biological Theory, 1(4), 352-356.

Barbieri, M. (2008). Biosemiotics: a new understanding of life. Naturwissenschaften, 95, 577-599.

Deely, J. (2006). On 'semiotics' as naming the doctrine of signs. Semiotica, 158, 1-33.

Favareau, D. (2007). The evolutionary history of biosemiotics. In M. Barbieri (Ed.), Introduction to biosemiotics (pp. 1-67). Dordrecht: Springer.

Frege, G. (1892). Über Sinn und Bedeutung. Zeitschrift fürPhilosophie und philosophische Kritik, 100, 25-50 . English translation in P. Geach and M. Black (eds.), Translations from the philosophical writings of Gottlob Frege. Oxford: Blackwell, 1952.

Krampen, M. (1981). Phytosemiotics. Semiotica, 36(3/4), 187-209.

Peirce, C. S. (1906). The basis of pragmaticism. In C. Hartshorne, \& P. Weiss (Eds.), The collected papers of Charles Sanders Peirce, vol. I-IV, (pp. 1931-1935). Cambridge, MA: Harvard University Press (Cited as CP followed by volume and paragraph number).

Posner, R., Robering, K., \& Sebeok, T. (1997). Semiotik/Semiotics: A handbook on the sign-theoretical foundations of nature and culture, vol. 1, (p. 4). Berlin: Walter de Gruyter.

Prodi, G. (1988). Material bases of signification. Semiotica, 69(3/4), 191-241.

Sebeok, T. (1963). Communication among social bees; porpoises and sonar; man and dolphin. Language, $39,448-466$.

Sebeok, T. (2001). Biosemiotics: Its roots, proliferation, and prospects. Semiotica, 134(1/4), 61-78. In K. Kull (ed.), Jakob von Uexküll: A paradigm for biology and semiotics.

Sonea, S. (1988). The global organism: a new view of bacteria. The Sciences, 28(4), 38-45.

Taborsky, E. (1999). Semiosis: the transformation of energy into information. Semiotica, 127, 599-646.

Taborsky, E. (2002). Energy and evolutionary semiosis. Sign Systems Studies, 30(1), 361-381. 